\title{
COMPARATIVE STUDY OF SIMPLE TRUSS SOLVED ACCORDING TO THE THEORIES OF 1ST AND 2ND ORDER
}

\author{
K. Frydrýšek ${ }^{*}$ M. Jahodová**, Z. Morávkováa**
}

\begin{abstract}
This contribution deals with the simple planar asymmetric pin-connected truss with 3 members. The ways and methods of derivations and solutions according to theories of first and second order and other possible linearizations are shown (i.e. internal forces, reactions, elongations, stresses). There are applied linear and nonlinear approaches and their simplifications via Taylor's series. Finally, the errors of all approaches are evaluated and compared.
\end{abstract}

Keywords: Planar truss, theories of first and second order, nonlinearities, error estimations, numerical methods.

\section{Introduction}

Planar (i.e. 2D) truss structures appear to be the easiest ways of introducing, explaining and solving geometrical and material nonlinearities; see Bažant and Cedolin (1991) and Frydrýšek (2016). In mechanics, for small deformations, tasks of this type (displacements, strains and stresses etc.) can be solved according to the simple first order (linear) theory or the more precise but more demanding second order (nonlinear) theory. If the effects of deformations of the structure under loadings are negligible with respects to the equilibrium of external and internal forces, first order analysis can be applied. The second order theory usually leads to a nonlinear equation which can be solved via several numerical methods. However, there are some possibilities for simplifying it, for example via Taylor's series etc.

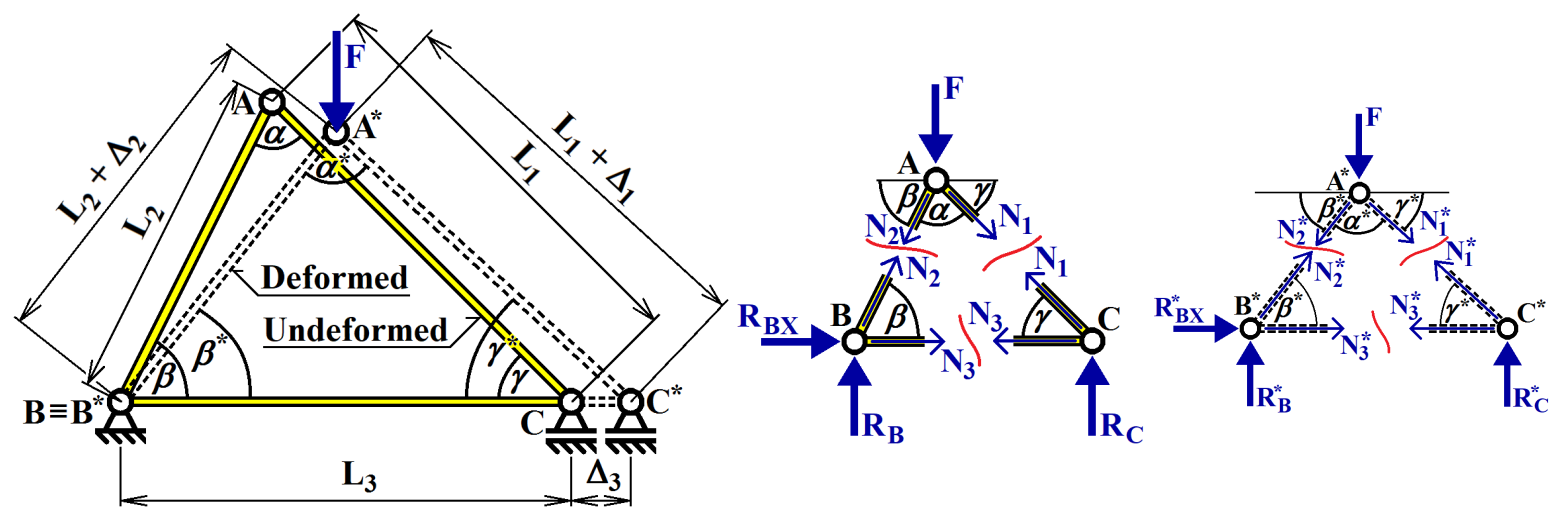

(a) Deformed and undeformed truss

(b) Theory of first order

(c) Theory of second order

Fig. 1: Simple (2D) statically determinate pin-connected truss.

This article presents the solution of a simple (2D) statically determinate pin-connected truss consisting of three members, see Fig. 1a (i.e. the derivation according to the first and second order theories, possible

Assoc. Prof. M.Sc. Karel Frydrýšek, Ph.D.: Department of Applied Mechanics, Faculty of Mechanical Engineering ; VSB Technical University of Ostrava; 17. listopadu 2172/15; 708 00, Ostrava - Poruba; CZ, karel.frydrysek@ @sb.cz

** Mgr. Monika Jahodová, Ph.D.: Department of Mathematics and Descriptive Geometry, Faculty of Mechanical Engineering; VSB - Technical University of Ostrava; 17. listopadu 2172/15; 708 00, Ostrava - Poruba; CZ, monika.jahodova@ vsb.cz

*** Mgr. Zuzana Morávková, Ph.D.: Department of Mathematics and Descriptive Geometry, Faculty of Mechanical Engineering; VSB - Technical University of Ostrava; 17. listopadu 2172/15; 708 00, Ostrava - Poruba; CZ, zuzana.moravkova@ vsb.cz 
simplifications, ways of solution, error estimation). The truss is loaded by the vertical force F. In the mechanics of truss structures, there is a lack of information about error estimation of the theory of first order, therefore we deal with this problems in this article.

\section{Solution According to the Theories of First and Second Orders}

Expressions are derived for the normal forces $\mathrm{N}_{i}[\mathrm{~N}]$, the axial stresses $\sigma_{i}[\mathrm{~Pa}]$, the elongations $\Delta_{i}[\mathrm{~m}]$ in all members $i=1,2$ and 3 and the reactions $\mathrm{R}_{\mathrm{BX}}, \mathrm{R}_{\mathrm{B}}, \mathrm{R}_{\mathrm{C}}$, see Fig. 1 , according to the theory of the small deformations for first and second order analyses, see Tab. 1 and 2. The given inputs are the force F, length of the members $\mathrm{L}_{i}[\mathrm{~m}]$, the modulus of elasticity $\mathrm{E}_{i}[\mathrm{~Pa}]$ of the material of the members, area of the cross-sections $\mathrm{A}_{i}\left[\mathrm{~m}^{2}\right]$ of the members. The angles $\alpha, \beta$ and $\gamma$ are explained in Fig.1. The results of the theory of second order is noted by upper subscript "*".

Tab. 1: Derived expressions and solution - theory of first order (direct linear solution based on simple linear statics); see Fig. 1(a,b).

$$
\begin{aligned}
& \Delta_{i}=\frac{\mathrm{N}_{i} \mathrm{~L}_{i}}{k_{i}} \text { for } i=1,2,3 \text { where } k_{i}=\mathrm{E}_{i} \mathrm{~A}_{i} \quad \mathrm{R}_{\mathrm{B}}=\frac{\mathrm{FL}_{1} \cos \gamma}{\mathrm{L}_{3}} \quad \mathrm{R}_{\mathrm{C}}=\frac{\mathrm{FL}_{2} \cos \beta}{\mathrm{L}_{3}} \quad \mathrm{R}_{\mathrm{BX}}=0 \\
& \mathrm{~N}_{1}=\frac{-\mathrm{F}}{\sin \gamma+\cos \gamma \cdot \tan \beta} \quad \mathrm{N}_{2}=\frac{-\mathrm{F}}{\sin \beta+\cos \beta \cdot \tan \gamma} \quad \mathrm{N}_{3}=\frac{\mathrm{F}}{\tan \beta+\tan \gamma}
\end{aligned}
$$

Tab. 2: Derived expressions - theory of second order (nonlinear solution); see Fig. 1(a,c).

$$
\begin{aligned}
& \Delta_{i}^{*}=\frac{\mathrm{N}_{i}^{*} \mathrm{~L}_{i}}{k_{i}} \text { for } i=1,2,3 \text { where } k_{i}=\mathrm{E}_{i} \mathrm{~A}_{i} \\
& \mathrm{~N}_{1}^{*}=\frac{-\mathrm{F}}{\sin \gamma^{*}+\cos \gamma^{*} \cdot \tan \beta^{*}} \quad \text { Law of sines: } \frac{\mathrm{L}_{1}+\Delta_{1}^{*}}{\sin \beta^{*}}=\frac{\mathrm{L}_{3}+\Delta_{3}^{*}}{\sin \alpha^{*}} \quad \mathrm{R}_{\mathrm{B}}{ }^{*}=\frac{\mathrm{F}\left(\mathrm{L}_{1}+\Delta_{1}^{*}\right) \cos \gamma^{*}}{\mathrm{~L}_{3}+\Delta_{3}^{*}} \\
& \mathrm{~N}_{2}^{*}=\frac{-\mathrm{F}}{\sin \beta^{*}+\cos \beta^{*} \cdot \tan \gamma^{*}} \quad \text { Law of sines: } \frac{\mathrm{L}_{1}+\Delta_{1}^{*}}{\sin \beta^{*}}=\frac{\mathrm{L}_{2}+\Delta_{2}^{*}}{\sin \gamma^{*}} \quad \mathrm{R}_{\mathrm{C}}{ }^{*}=\frac{\mathrm{F}\left(\mathrm{L}_{2}+\Delta_{2}^{*}\right) \cos \beta^{*}}{\mathrm{~L}_{3}+\Delta_{3}^{*}} \\
& \mathrm{~N}_{3}^{*}=\frac{\mathrm{F}}{\tan \beta^{*}+\tan \gamma^{*}} \quad \text { Triangle rule: } \alpha^{*}+\beta^{*}+\gamma^{*}=\pi \quad \mathrm{R}_{\mathrm{BX}}{ }^{*}=0
\end{aligned}
$$

\section{The Systems of Governing Equations and the Applied Numerical Methods}

The first order theory leads to a set of linear equations the solution of which is presented in Tab. 1 . We use relations for $\Delta_{i}^{*}, \mathrm{~N}_{\mathrm{i}}^{*}, i=1,2,3$, both laws of sines and the triangle rule from Tab. 2 (theory of second order) and we obtain the system of six nonlinear equations for unknown deformation parameters $\Delta_{1}^{*}, \Delta_{2}^{*}$, $\Delta_{3}^{*}, \alpha^{*}, \beta^{*}, \gamma^{*}$. Let's denote the left sides of this system as functions $f_{i}$ for $i=1, \ldots, 6$ and the vector of unknowns $\boldsymbol{x}=\left(\Delta_{1}^{*}, \Delta_{2}^{*}, \Delta_{3}^{*}, \alpha^{*}, \beta^{*}, \gamma^{*}\right)^{\top}$.

$$
\left.\begin{array}{r}
\Delta_{1}^{*}\left(\tan \beta^{*} \cos \gamma^{*}+\sin \gamma^{*}\right)+\mathrm{FL}_{1} / k_{1}=0 \\
\Delta_{2}^{*}\left(\tan \gamma^{*} \cos \beta^{*}+\sin \beta^{*}\right)+\mathrm{FL}_{2} / k_{2}=0 \\
\Delta_{3}^{*}\left(\tan \beta^{*}+\tan \gamma^{*}\right)-\mathrm{FL}_{3} / k_{3}=0 \\
\Delta_{1}^{*} \sin \gamma^{*}-\Delta_{2}^{*} \sin \beta^{*}+\mathrm{L}_{1} \sin \gamma^{*}-\mathrm{L}_{2} \sin \beta^{*}=0 \\
\Delta_{1}^{*} \sin \alpha^{*}-\Delta_{3}^{*} \sin \beta^{*}+\mathrm{L}_{1} \sin \alpha^{*}-\mathrm{L}_{3} \sin \beta^{*}=0 \\
\alpha^{*}+\beta^{*}+\gamma^{*}-\pi=0
\end{array}\right\} f_{i}(\boldsymbol{x})=0 \text { for } i=1, \ldots, 6 \Leftrightarrow \boldsymbol{f}(\boldsymbol{x})=\mathbf{0}
$$

The system (1) we solve by the another type of linearization and by numerical methods for nonlinear equations.

For the linearization we approximated left sides of equations in the system (1) by Taylor series in several variables of order one. For example, we get the following approximation from the first equation: 


$$
\begin{aligned}
& \Delta_{1}^{*}\left(\tan \beta^{*} \cos \gamma^{*}+\sin \gamma^{*}\right)+\mathrm{FL}_{1} / k_{1} \approx \Delta_{1}(\tan \beta \cos \gamma+\sin \gamma)+\mathrm{FL}_{1} / k_{1}+ \\
& +\Delta_{1} \frac{1}{\cos ^{2} \beta} \cos \gamma\left(\beta-\beta^{*}\right)+\Delta_{1}(-\tan \beta \sin \gamma+\cos \gamma)\left(\gamma-\gamma^{*}\right)+(\tan \beta \cos \gamma+\sin \gamma)\left(\Delta_{1}-\Delta_{1}^{*}\right)
\end{aligned}
$$

and we obtain system of six linear equations

$$
\begin{gathered}
\left(\begin{array}{cccccc}
\sin (\beta+\gamma) & 0 & 0 & 0 & \Delta_{1} \frac{\cos \gamma}{\cos \beta} & \Delta_{1} \cos (\beta+\gamma) \\
0 & \sin (\beta+\gamma) & 0 & 0 & \Delta_{2} \cos (\beta+\gamma) & \Delta_{2} \frac{\cos \beta}{\cos \gamma} \\
0 & 0 & \sin (\beta+\gamma) & 0 & \Delta_{3} \frac{\cos \gamma}{\cos \beta} & \Delta_{3} \frac{\cos \beta}{\cos \gamma} \\
\sin \gamma & -\sin \beta & 0 & 0 & -\left(\Delta_{2}+\mathrm{L}_{2}\right) \cos \beta & \left(\Delta_{1}+\mathrm{L}_{1}\right) \cos \gamma \\
\sin \alpha & 0 & -\sin \beta & \left(\Delta_{1}+\mathrm{L}_{1}\right) \cos \alpha & -\left(\Delta_{3}+\mathrm{L}_{3}\right) \cos \beta & 0 \\
0 & 0 & 0 & 1 & 1
\end{array}\right) \cdot\left(\begin{array}{c}
\Delta_{1}^{*} \\
\Delta_{2}^{*} \\
\Delta_{3}^{*} \\
\alpha^{*} \\
\beta^{*} \\
\gamma^{*}
\end{array}\right)= \\
\left(\begin{array}{c}
2 \Delta_{1} \sin (\beta+\gamma)+\Delta_{1} \beta \frac{\cos \gamma}{\cos \beta}+\Delta_{1} \gamma \cos (\beta+\gamma)+\mathrm{F} \frac{\mathrm{L}_{1}}{k_{1}} \cos \beta \\
\Delta_{2} \sin (\beta+\gamma)+\Delta_{2} \gamma \frac{\cos \beta}{\cos \gamma}+\Delta_{2} \beta \cos (\beta+\gamma)+\mathrm{F} \frac{\mathrm{L}_{2}}{k_{2}} \cos \gamma \\
\left(\Delta_{1}+\mathrm{L}_{1}\right)(\sin \gamma+\gamma \cos \gamma)-\left(\Delta_{2}+\mathrm{L}_{2}\right)(\sin \beta+\beta \cos \beta)+\sin \gamma \Delta_{1}-\sin \beta \Delta_{2} \\
\left(\Delta_{1}+\mathrm{L}_{1}\right)(\sin \alpha+\alpha \cos \alpha)-\left(\Delta_{3}+\mathrm{L}_{3}\right)(\sin \beta+\beta \cos \beta)+\sin \alpha \Delta_{1}-\sin \beta \Delta_{3}
\end{array}\right)
\end{gathered}
$$

The system of linear equations (2) is nearly similar to the theory of first order written in Tab. 1. For more informations see Frydrýšek (2016).

The system (1) is solved by well known Newton method and by Broyden method. The Broyden method for solving the system of nonlinear equations is based on the secant method, see Quarteroni (2006). The initial approximation $\boldsymbol{x}^{0}=(0,0,0, \alpha, \beta, \gamma)^{\top}$ is choosen. Lets denote the initial approximation of Jacobian matrix as

$$
\mathbf{Q}^{0}=\left(\begin{array}{cccccc}
\tan \beta \cos \gamma+\sin \gamma & 0 & 0 & 0 & 0 & 0 \\
0 & \tan \gamma \cos \beta+\sin \beta & 0 & 0 & 0 & 0 \\
0 & 0 & \tan \beta+\tan \gamma & 0 & 0 & 0 \\
\sin \gamma & -\sin \beta & 0 & 0 & -\mathrm{L}_{2} \cos \beta & \mathrm{L}_{1} \cos \gamma \\
\sin \alpha & 0 & -\sin \beta & \mathrm{L}_{1} \cos \alpha & -\mathrm{L}_{3} \cos \beta & 0 \\
0 & 0 & 0 & 1 & 1 & 1
\end{array}\right)
$$

The next approximations $\boldsymbol{x}^{k}$ are computed in following three steps for $k=1, \ldots$

1. step: Find $\boldsymbol{d}^{k}$ as the solution of the system of linear equation $\mathbf{Q}^{k-1} \boldsymbol{d}^{k}=-\boldsymbol{f}\left(\boldsymbol{x}^{k-1}\right)$.

2. step: Compute the next approximation $\boldsymbol{x}^{k}=\boldsymbol{x}^{k-1}+\boldsymbol{d}^{k}$.

3. step: Compute the next matrix $\mathbf{Q}^{k}=\mathbf{Q}^{k-1}+\frac{\boldsymbol{f}\left(\boldsymbol{x}^{k-1}\right) \cdot \boldsymbol{d}^{k^{\top}}}{\boldsymbol{d}^{k^{\top}} \cdot \boldsymbol{d}^{k}}$.

Numerical results are calculated for inputs written in Tab. 3. Newton and Broyden iterative approaches converged to the same results.

\section{Conclusions}

It is a fact that the planar truss structures appear to be the easiest ways of introducing, explaining and solving geometrical and material nonlinearities (in this case, a simple pin-connected and statically determinate truss of three members with variable length $L_{1}$ ). The focus is on the theory of first and second order, understanding, derivation and solution of linear/nonlinear problems and error estimation. Hence, from calculated results, it is obvious the legitimacy application of the theory of second order which gives results close to the reality. Some mentioned errors of the theory of first order are alarming for small length $\mathrm{L}_{1}$ or for obtuse angles or in cases of buckling, see Fig. 2 and Tab. 3. 


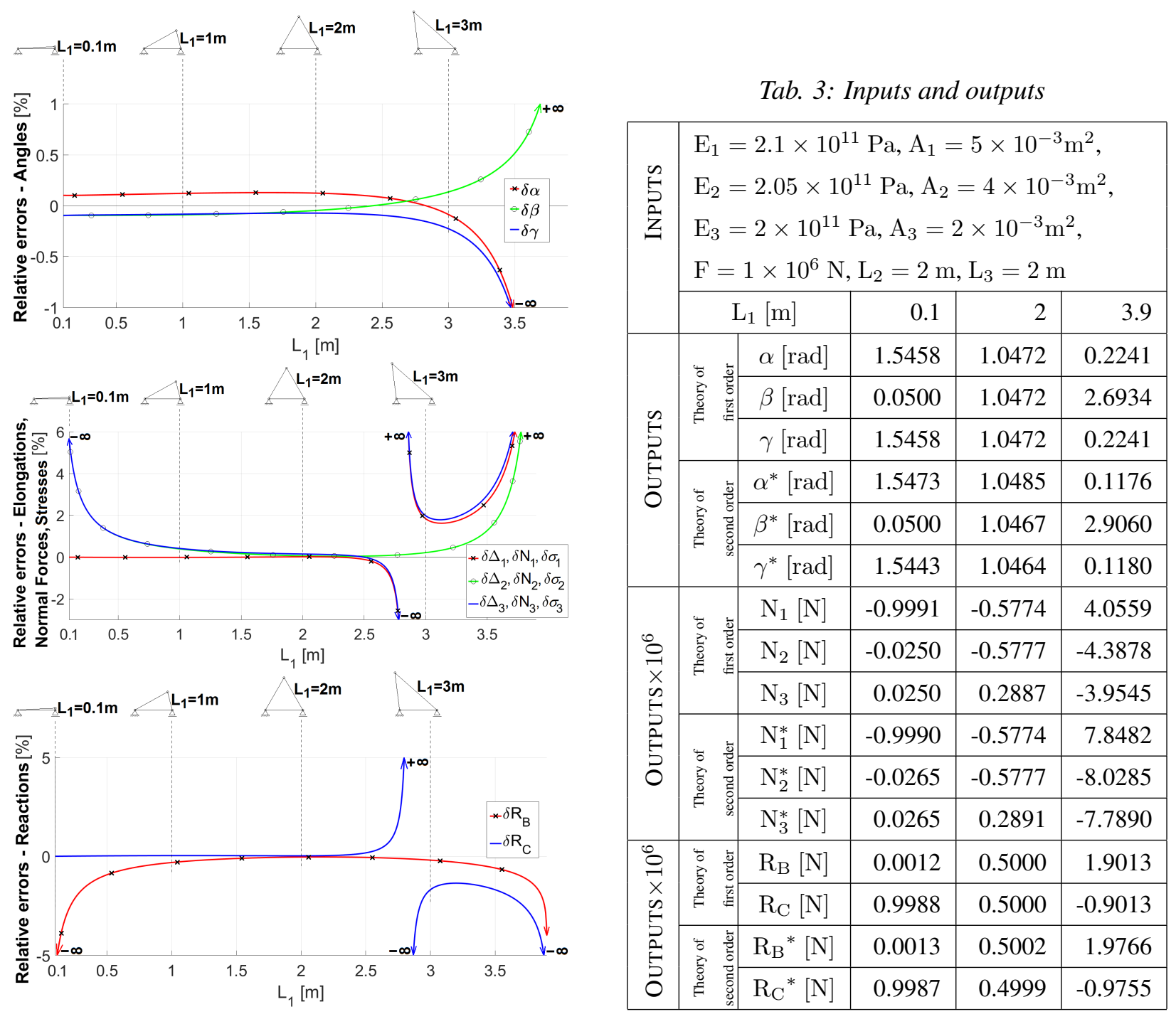

Fig. 2: Relative errors

However, there is not enough information about error estimations and comparative studies for the theories of first and second order. Hence, this article fills the lack of information and gives to engineers some basic ideas/concepts about possible errors/mistakes. There are other comparative studies of truss structures based on the theory of probability and Monte Carlo Method too, see Frydrýšek and Jančo (2016).

\section{Acknowledgments}

This article was supported by the project No. CZ.02.1.01/0.0/0.0/17_049/0008441 within the Operational Programme Research, Development and Education financed by the European Union and from the state budget of the Czech Republic and by the Czech project SP2019/100.

\section{References}

Bažant, Z.P., Cedolin, L. (1991) Stability of Structures: Elastic, Inelastic, Fracture and Damage Theories. Oxford University Press, New York, 3rd ed., 2010, pp. 1-1011.

Frydrýšek, K. (2016) Some Selected Tasks of Elasticity and Plasticity 4 (Basic Nonlinear Mechanics of Deformable Bodies in Examples). Department of Applied Mechanics, Faculty of Mechanical Engineering, VSB - Technical University of Ostrava, Ostrava, pp. 1-139, ISBN 978-80-248-4152-6.

Frydrýšek, K., Jančo, R. (2016) Simple planar truss (linear, nonlinear and stochastic approach. Journal of Mechanical Engineering, 66, 2, pp 5-12, ISSN 00392472, doi:10.1515/scjme-2016-0013.

Quarteroni, A., Sacco, R., Saleri, F. (2006) Numerical Mathematics (Texts in Applied Mathematics 37). Springer, New York, pp.281-294. 